\title{
Estudo dos nichos ecológicos de leveduras patogênicas das espécies Cryptococcus neoformans e Cryptococcus gattii na cidade de Londrina, PR
}

\section{Study of ecological niches from pathogenic yeasts of the species Cryptococcus neoformans and Cryptococcus gattii in Londrina City, PR}

\author{
Aline Artioli Machado Yamamura ${ }^{1 *}$; Roberta Lemos Freire ${ }^{2}$; \\ Milton Hissashi Yamamura ${ }^{3}$; Aline Felix ${ }^{4}$; Alessandra Taroda ${ }^{5}$
}

\begin{abstract}
Resumo
Os fungos patogênicos das espécies Cryptococcus neoformans e Cryptococcus gattii podem ser encontrados no meio ambiente em guano de pombos, solo com excrementos de pombos e pássaros, além de matéria vegetal de árvores. O objetivo deste estudo foi investigar a presença das espécies patogênicas de Cryptococcus em solo com excrementos de pombos, solo com matéria vegetal e em madeira em decomposição de partes ocas de árvores existentes em lugares públicos da cidade de Londrina, PR. Trinta locais foram selecionados dentre praças, parques e ruas da região central da cidade onde amostras foram coletadas de: solo com excretas de pombos $(\mathrm{n}=120)$, solo com matéria vegetal da base das árvores $(\mathrm{n}=120)$ e $s w a b$ de matéria vegetal em decomposição de partes ocas de árvores, no período de junho de 2009 a março de 2010. As coletas abrangeram três estações do ano: inverno, primavera e verão, nesse período 360 amostras foram obtidas e analisadas em quatro diferentes etapas. As espécies foram identificadas por meio de análise microscópica da cultura e crescimento em meios seletivos. Um total de cinco amostras foi positivo para Cryptococcus spp. (1,38\%), três amostras foram identificadas como C. neoformans $(0,84 \%)$, duas amostras de solo com excrementos de pombos e uma amostra vegetal do solo. As amostras eram provenientes de um parque, uma rua e uma praça localizados na área central de Londrina. C. gattii foi isolado das outras duas amostras ( $0,54 \%)$, uma em solo com excrementos de pombos e outra em amostra de matéria vegetal do solo obtida de dois parques da cidade localizados em área desprotegida contra insolação e chuvas. Não foi obtido isolamento de Cryptococcus spp. das 120 amostras de $s w a b$ de madeira em decomposição de partes ocas das 12 espécies de árvores amostradas. Os resultados das análises do meio ambiente confirmaram a ocorrência das espécies patogênicas Cryptococcus neoformans e Cryptococcus gattii em locais onde a população suscetível de humanos e animais podem ser expostos aos propágulos infecciosos dos fungos.
\end{abstract}

Palavras-chave: Pombos, leveduras, ecologia dos fungos, Cryptococcus spp., Criptococose

\footnotetext{
1 Prof ${ }^{\mathrm{a}}$. de Microbiologia Veterinária, Dept ${ }^{\mathrm{o}}$ de Medicina Veterinária Preventiva, Universidade Estadual de Londrina, Centro de Ciências Agrárias, UEL, Londrina, PR. E-mail: alineartioli@uel.br

2 Prof ${ }^{a}$. de Epidemiologia Aplicada à Medicina Veterinária, Dept ${ }^{\circ}$ de Medicina Veterinária Preventiva, UEL/CCA, Londrina, PR. E-mail: rlfreire@uel.br

3 Prof. de Parasitologia Veterinária, Dept ${ }^{\circ}$ de Medicina Veterinária Preventiva, UEL/CCA, Londrina, PR. E-mail: yamamura@uel. br

4 Especialização em Residência de Moléstias Infecciosas, Dept ${ }^{\circ}$ de Medicina Veterinária Preventiva, UEL/CCA, Londrina, PR. E-mail: alinefelix.lih@hotmail.com

5 Especialização em Residência de Moléstias Parasitárias, Dept ${ }^{\circ}$ de Medicina Veterinária Preventiva, UEL/CCA, Londrina, PR. E-mail: ataroda@terra.com.br

* Autor para correspondência
} 


\begin{abstract}
The pathogenic fungi of the species Cryptococcus neoformans and Cryptococcus gattii could be found in the environment on pigeon guano, soil with pigeon and bird excreta and vegetable material from trees. The aim of this study was analyze the presence of the Cryptococcus pathogenic species on soil with pigeon excreta, soil with vegetable material and decayed wood of trunk hollows of trees from public places in the city of Londrina, PR. Thirty public places were selected from squares, parks and streets in the city centre where samples were collected from: soil with pigeon excreta $(n=120)$, soil with vegetable material $(n=120)$ and decayed wood of trunk hollows of trees $(n=120)$, during June 2009 until March 2010. These collect comprised the seasons: winter, spring and summer, during this period 360 samples were obtained and analyzed in four different times. The species were identified by means of microscopic analysis from culture and their rising on selective medium. A total of five samples were positive to Cryptococcus spp. $(1,38 \%)$ three were positive to Cryptococcus neoformans $(0,84 \%)$ in two samples of soil with pigeon excreta, the other one on soil with vegetable material. The samples were originated from a park, a street and a square, placed in the city centre area of Londrina. Cryptococcus gattii was isolated from two samples $(0,54 \%)$, one in soil with pigeon excreta and another in vegetable material obtained from the soil of two parks that were not protected against sunshine and rain. Cryptococcus spp. was not isolated in none of the 120 swabs samples of decayed wood trunk hollows from the 12 species of trees sampled. The results of the analyses from the environment confirmed the occurrence of the pathogenic species Cryptococcus neoformans e Cryptococcus gattii in places were the human and animal susceptible populations could be exposure to infectious fungi propagators.
\end{abstract}

Key words: Pigeon, yeasts, fungi ecology, Cryptococcus spp., Cryptococcosis

\section{Introdução}

Cryptococcus neoformans e Cryptococcus gattii são fungos patogênicos que causam doença em várias espécies animais, incluindo mamíferos placentários terrestres, marsupiais, cetáceos, aves e répteis. Estas espécies de Cryptococcus causam a criptococose doença fúngica oportunista, localizada ou disseminada, que atinge os pulmões tornandose muitas vezes fatal em humanos, principalmente, quando afeta o sistema nervoso central (KWONCHUNG et al., 2000; LACAZ et al., 2002).

Historicamente, C. neoformans (Sanfelice) Vuillemin, 1901, foi classificado em duas variedades e cinco sorotipos: Cryptococcus neoformans var. neoformans (sorotipos A, D e AD) e, Cryptococcus neoformans var. gattii (sorotipos $\mathrm{B}$ e C). Devido à evidência molecular (FRANZOT; SALKIN; CASADEVALL, 1999; BOEKHOUT et al., 2001) passou-se a adotar a nomenclatura revisada e atualizada por Kwon-Chung et al. (2002). As leveduras foram re-classificadas como duas espécies, C. neoformans e C. gattii (KWONCHUNG et al., 2002) que diferem quanto à epidemiologia, apresentação clínica e terapêutica (KWON-CHUNG; BENNETT, 1992; SPEED; DUNT, 1995; SORREL, 2001). O agente patogênico predominantemente oportunista $C$. neoformans possui duas variedades $C$. neoformans var. grubii (sorotipos A e AD) e C. neoformans var. neoformans (sorotipo D) e o agente patogênico primário $C$. gattii (sorotipos B e C) com base em análises dos padrões genéticos do DNA (FRANZOT; SALKIN; CASADEVALL, 1999).

C. neoformans é cosmopolita, do ponto de vista epidemiológico, enquanto $C$. gattii é limitado a certas regiões tropicais. A criptococose é adquirida pela inalação de propágulos infecciosos a partir de uma fonte ambiental. C. neoformans é encontrado na natureza, geralmente em fezes de pombos, outras aves e em árvores, pode viver saprofíticamente no organismo de humanos e dos animais domésticos e silvestres (criptococose-infecção) (ELLIS; PFEIFFER, 1990; LAZÉRA et al., 1996). C. gattii encontra-se presente em folhas, partes ocas do tronco e madeira em decomposição de árvores pertencentes às espécies australianas Eucalyptus camaldulensis e E. tereticornis, além de árvores nativas de 
espécies existentes em regiões dos continentes de clima tropical e subtropical (SORREL, 2001; RANDHAWA; KOWSHIK; KHAN, 2003). As diferentes características epidemiológicas dos fungos das espécies patogênicas do gênero Cryptococcus determinam seja necessário o estudo do habitat, das variações sazonais em relação à produção de propágulos infecciosos e das vias de transmissão, desse modo medidas de profilaxia e controle da infecção poderão ser desenvolvidas para humanos e animais (RESTREPO et al., 2000).

O objetivo deste estudo foi investigar a ocorrência de fungos das espécies patogênicas de Cryptococcus em seu habitat ou nicho ecológico no meio ambiente dos lugares públicos da cidade de Londrina, PR, por meio da pesquisa do solo com excrementos de pombos, solo com matéria vegetal de árvores e madeira em decomposição do tronco de árvores.

\section{Material e Métodos}

A área de estudo, localização geográfica e condições climáticas do município de Londrina correspondem aos seguintes dados: $23^{\circ} 08^{\prime} 47^{\prime \prime} \mathrm{S}$ e $23^{\circ} 35^{\prime} 08^{\prime}$ 'S de latitude e longitude $50^{\circ} 59^{\prime} 50^{\prime}$ 'W e 5055'49"W, altitude máxima $690 \mathrm{~m}$, altitude mínima $425 \mathrm{~m}$ acima do nível do mar, clima subtropical úmido com chuvas em todas as estações, temperatura média anual de $21^{\circ} \mathrm{C}$, temperatura média máxima de $27,6^{\circ} \mathrm{C}$, temperatura média mínima de $15,8^{\circ} \mathrm{C}$ e índices de umidade relativa do ar média anual de 70-75\% (PREFEITURA DO MUNICÍPIO DE LONDRINA, 2010).

O tamanho de amostra para a população alvo a ser estudada foi determinado utilizando-se o pacote estatístico EPI6 (CDC - Atlanta). Para uma população infinita, adotou-se a prevalência esperada de $50 \%$, erro de $5 \%$ e nível de significância de $5 \%$, resultando em 360 amostras. A adoção de 50\% de prevalência esperada é uma pratica comum quando não há estudos que demonstrem a prevalência na região, dessa forma minimiza-se o erro de amostragem e garante-se a precisão da pesquisa.

A região central da cidade de Londrina e os bairros contíguos possuem arborização em todas as ruas e praças, fato que permite o assentamento de pombos e conseqüente queda e acúmulo dos excrementos em calçadas e ruas. Dentro dessa área foram selecionados 30 locais protegidos e não protegidos (ruas, parques e praças) altamente contaminados com dejetos de pombos e próximos a ruas movimentadas ou a um fluxo significativo de pessoas. No período de Junho de 2009 a Março de 2010 foram colhidas e analisadas 360 amostras dos 30 locais selecionados, da seguinte forma: 30 locais x três unidades amostrais x quatro ciclos de coleta. A unidade de amostragem consistiu de: (i) Swabs de matéria vegetal existente na madeira em decomposição de partes ocas do tronco de árvores $(n=120)$, (ii) solo com excrementos de pombos $(\mathrm{n}=120)$, e (iii) matéria vegetal presente no solo da base das árvores (detritos de folhas e madeira de árvores) $(\mathrm{n}=120)$.

As amostras de solo com excrementos de pombos e com matéria vegetal foram colhidas utilizando-se espátulas estéreis individuais e acondicionadas em placas de petri esterilizadas, em duplicata, e em quantidade aproximada de 4,0 a 20 g (MONTENEGRO; PAULA, 2000). As amostras (swabs e amostras de solo) foram transportadas em caixas isotérmicas, mantidas a $4^{\circ} \mathrm{C}$, processadas e analisadas no Laboratório de Micologia Veterinária da Universidade Estadual de Londrina.

Os swabs das amostras foram processados num período de duas horas após a coleta, da seguinte forma: $10 \mathrm{ml}$ de solução fisiológica estéril contendo gentamicina $(25 \mathrm{mg} / \mathrm{l})$ foram adicionados em cada tubo de ensaio contendo um swab, seguido de turbilhonamento por 2 minutos em agitadora, após o descarte dos swabs a suspensão do conteúdo dos tubos foi retirada após sedimentação por 30 minutos e alíquotas de $100 \mu$ foram semeadas em placas de agar Sabouraud dextrose, incubadas em temperatura de $32^{\circ} \mathrm{C}$, num período de dez dias até a formação de 
colônias e o exame microscópico (RANDHAWA; KOWSHIK; KHAN, 2005).

As amostras do solo com detritos da matéria vegetal e excrementos de pombos foram processadas em 48 horas após a coleta. Foram adicionados $30 \mathrm{ml}$ de solução salina esterilizada com adição de cloranfenicol e ampicilina (150 mg/ml cada) a 2,0 g de cada um dos tipos de amostras. Após homogeneização, cada uma das amostras foi filtrada em gaze esterilizada e uma alíquota de $100 \mu \mathrm{l}$ semeada em ágar Sabouraud dextrose. As culturas foram incubadas a $35^{\circ} \mathrm{C}$ e examinadas diariamente durante 21 dias (MONTENEGRO; PAULA, 2000; RIBEIRO et al., 2006).

A identificação do gênero Cryptococcus teve como base a morfologia macroscópica das colônias com aspecto leveduriforme em agar Sabouraud dextrose, o isolamento seletivo em meio de Staib ou agar semente de niger (Guizotia abyssinica), a morfologia microscópica pela coloração com tinta nanquim e o teste de uréase (LACAZ et al., 1998). Para a identificação das espécies foi utilizado teste de crescimento em agar CGB (canavanina-glicinaazul-de-bromotimol) (LACAZ et al., 1998; MELO; LACAZ; CHARBELL, 1993; RIBEIRO et al., 2006).

\section{Resultados e Discussão}

Das 360 amostras analisadas cinco (1,38\%) foram positivas para Cryptococcus spp.. Destas, três amostras $(0,84 \%)$ apresentaram C. neoformans, duas em solo com excrementos de pombos e uma em solo com material vegetal das árvores; provenientes de parque, praça e rua (Figura 1). Leveduras do gênero Candida também foram isoladas nessas amostras
(Tabela 1). C. gattii foi isolado em duas amostras $(0,54 \%)$, uma de solo com excrementos de pombos e outra de solo com matéria vegetal e provinham de dois parques públicos, nominados "Zerão" e "Bosque" (Tabela 1; Figura 1). As duas amostras foram colhidas de áreas desprotegidas, expostas a radiação solar e chuvas constantes, em locais de elevada passagem de transeuntes. Conforme observado, na maioria das praças visitadas havia numero elevado de pombos e dejetos e em conseqüência havia precárias condições de higiene.

As árvores encontradas na região pesquisada pertencem às espécies: Bauhinia forficata, Eucalyptus camaldulensis, Eucalyptus tereticornis, Lagerstcoemia indica, Allophylus edulis, Ocatea pulchella, Schinus terebinthifolia, Ceasalpinea peltophoroides, Ligusteum japonicum, Ficus microcarpa, Gurucaia sp, Moquilea tomentosa. As árvores de maior porte representadas por Eucalyptus sp localizavam-se nos parques e as demais nas ruas e praças. Não houve positividade em nenhuma das 120 amostras de $s w a b$ da madeira em decomposição de partes ocas do tronco de árvores (Tabela 1).

Os excrementos de aves têm sido associados ao C. neoformans em muitos estudos (CURRIE; FREUNDLICH, CASADEVALL, 1994; YAMAMOTO et al., 1995; VARMA et al., 1995; FRANZOT et al., 1997; DELGADO et al., 2005). C. neoformans tem uma distribuição mundial, tendo sido isolado de várias origens na natureza. É conhecido por sua associação com dejetos de pombos, porém o fungo pode ser encontrado em excretas de várias aves, tais como psitacídeos, passeriformes, columbiformes e falconiformes (CAFARCHIA et al., 2006), no solo, na madeira em decomposição e em vegetais e frutas. 
Tabela 1. Resultados de culturas fúngicas em 360 amostras de solo com excrementos de pombos, solo com material vegetal e $s w a b$ de partes ocas de árvores, colhidos em 30 locais centrais da cidade de Londrina, PR, de junho de 2009 a março de 2010.

\begin{tabular}{|c|c|c|c|c|}
\hline \multirow[b]{2}{*}{ Amostras } & \multirow[b]{2}{*}{$\begin{array}{l}\text { Positivos/Total } \\
\text { (\%) }\end{array}$} & \multicolumn{3}{|l|}{ Fungos } \\
\hline & & $\begin{array}{l}\text { Cryptococcus neoformans } \\
\mathrm{n}^{\mathrm{o}} \text { positivos }\end{array}$ & $\begin{array}{l}\text { Cryptococcus gatti } \\
\mathrm{n}^{\mathrm{o}} \text { positivos }\end{array}$ & $\begin{array}{l}\text { Candida spp } \\
\mathrm{n}^{\circ} \text { positivos }\end{array}$ \\
\hline $\begin{array}{l}\text { Solo com } \\
\text { Excrementos }\end{array}$ & $2 / 120(0,54)$ & S & F & 2 \\
\hline $\begin{array}{l}\text { Solo com } \\
\text { material vegetal }\end{array}$ & $3 / 120(0,84)$ & 1 & 2 & 1 \\
\hline Swab & $0 / 120(0,00)$ & 0 & 0 & 0 \\
\hline Total & $5 / 360(1,38)$ & 3 & 2 & 3 \\
\hline
\end{tabular}

Fonte: Elaboração dos autores.

Figura 1. Locais onde foram isolados Cryptococcus neoformans e Cryptococcus gattii em amostras de solo com excretas de pombos e com matéria vegetal, na região central da cidade de Londrina, Paraná, 2009 - 2010.

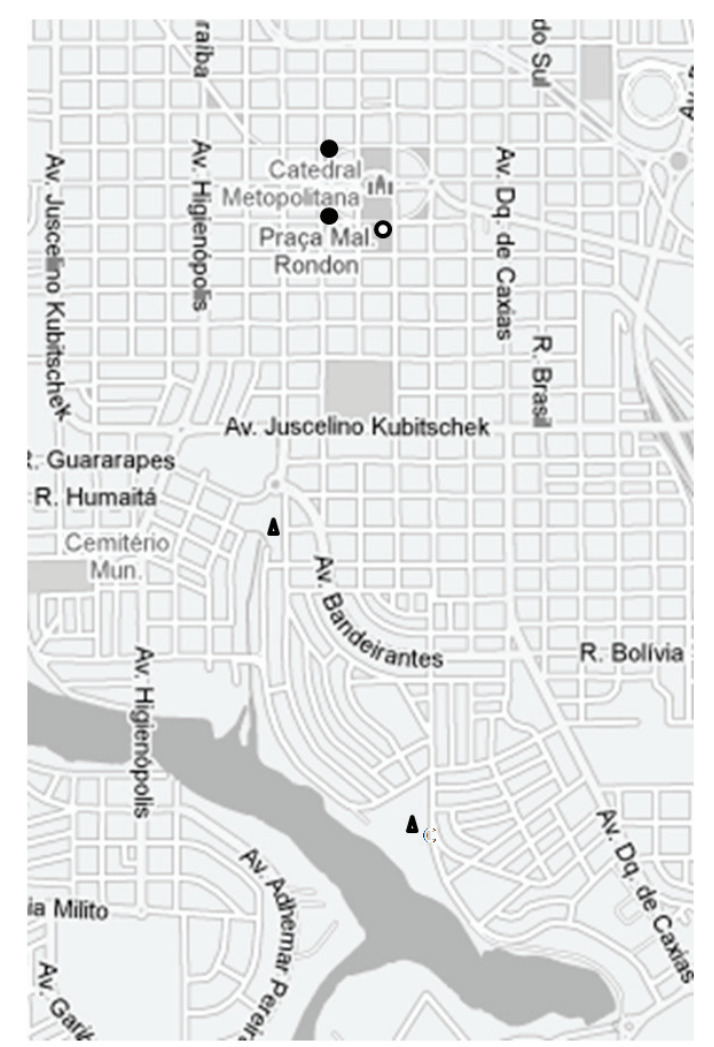

- Cryptococcus neoformans + Candida spp-Fezes

- Cryptococcus neoformans + Candida spp-Motéria Vegetal

ه Cryptococcus gattii-Motéria Vegetal

Fonte: Elaboração dos autores.

Centro da cidade de Londrina. Disponível em: $<$ https//www. Google/gps $>$

No Brasil foram pesquisados locais públicos, praças e parques do centro da cidade de São Paulo obtendo-se o isolamento de C. neoformans em $26,3 \%$ das amostras de excrementos de pombos com freqüência maior em locais protegidos do que desprotegidos (MONTENEGRO; PAULA, 2000). Locais desprotegidos favorecem a dessecação de excretas, porém C. neoformans é altamente 
resistente à dessecação. Esta característica favorece a sobrevivência do fungo quando ocorre a competição com outras populações microbianas presentes nos excrementos dos pombos (KWONCHUNG; BENNETT, 1992). A levedura seca, forma infectante, é facilmente propagada pelo ar (MITCHELL; PERFECT, 1995) e os excrementos de pombos com a presença de $C$. neoformans atuam como uma via natural de disseminação do fungo. Inversamente, em locais abertos e expostos raramente há a presença de $C$. neoformans, mesmo quando há grande acumulo de excrementos de pombos. Esta espécie de fungo apresenta baixa tolerância térmica, sendo muito suscetível à radiação solar. Esses fatores, possivelmente, limitam o desenvolvimento da levedura em excrementos de pombos de locais desprotegidos (ISHAQ; BULMER; FELTON, 1968; KWON-CHUNG; BENNETT, 1992).

Pesquisas dirigidas para matéria vegetal como previamente sugerido por Staib et al. (1972) e Bauwens et al. (1986) conduziram a novos achados ambientais que permitiram esclarecer a complexa ecologia de $C$. gattii. O papel que as árvores desempenhavam no ciclo de vida de $C$. gattii era pouco conhecido até que em 1990, Ellis e Pfeiffer, em estudo epidemiológico observaram a associação entre $C$. gattii sorotipo B e eucaliptos na Austrália, estabelecendo associação do nicho ecológico com: madeira em decomposição da base dos troncos de E. camaldulensis, folhas, flores onde os basidiósporos da fase sexuada são considerados propágulos infectantes dispersos no mesmo período de floração das árvores durante a primavera. Embora as espécies do Gênero Cryptococcus que pertencem ao Filo Basidiomycotina sejam encontradas no meio ambiente sob a forma sexuada de basidiósporos, nos tecidos dos hospedeiros esses microrganismos existem, exclusivamente, como leveduras encapsuladas com característica morfológica definida. Em conseqüência da distribuição da doença em humanos estar correlacionada, no início dos anos 1990, com os eucaliptos hospedeiros, a ampla pesquisa realizada pelos autores, acima citados, elaborou uma proposta de ligação epidemiológica entre as espécies de eucaliptos hospedeiras e a infecção humana causada por Cryptococcus gattii. Evidenciada pela alta incidência de colonização do vestíbulo nasal de coalas, animais da fauna da Austrália, com Cryptococcus gattii, pois as folhas de E. camaldulensis e E. tereticornis constituem numa fonte de alimento preferido desses animais e o tipo molecular isolado de eucaliptos corresponde ao isolado obtido de coalas, dos locais de colonização e/ou infecção, tais como: vestíbulo nasal, garras e pele (ELLIS; PFEIFFER, 1990).

Pesquisas confirmaram que além dos eucaliptos as árvores nativas de outros países poderiam ser consideradas reservatórios de $C$. gattii nas espécies existentes em regiões dos continentes de clima tropical e subtropical, tais como: Cassia grandis, Ficus microcarpa, Syzygium jambolana, Sygygium cumini, Terminalia catappa e Senna multijuga ((PFEIFFER; ELLIS, 1991; SORREL, 2001; RANDHAWA; KOWSHIK; KHAN, 2003). Devido à exportação comercial de Eucalyptus camaldulensis e E. tereticornis da Austrália, durante décadas, sendo que as espécies de eucaliptos têm sido estabelecidas em muitos países e regiões do globo terrestre, tais como: Estados Unidos (Havaí Califórnia,), sudoeste da Europa, região mediterrânea, subcontinente indiano, Américas do Sul e Central (Brasil, México), África (regiões central, sudoeste, sul), China e Papua Nova Guiné (SORREL, 2001). C. gattii sorotipo B e C. neoformans var. grubii (sorotipo A) costumam ser isoladas de flores e tronco de eucaliptos em diferentes regiões da India e de madeira em decomposição de troncos de árvores da espécie Syzygium cumini no noroeste da India (GUGNANI et al., 2005; RANDHAWA et al., 2006). Os estudos ambientais no Brasil tiveram início na região sudeste na cidade do Rio de Janeiro, em zona urbana, onde as amostras foram coletadas de madeira em decomposição do interior das partes ocas de troncos das árvores selecionadas (LAZÉRA; WANKE; NISHIKAWA, 1993; LAZÉRA et al., 1996). Nesses estudos foi isolado C. gattii em 
oito $(25,8 \%)$ de 31 espécies de árvores, tais como: ameixa de Java (Sygygium jambolanum), nativa da India e Brasil; chuva de novembro (Senna multijuga) proveniente da mata Atlântica; figueira (Ficus microcarpa) originária da Malásia, India e China, mas amplamente plantada como árvore ornamental; e chuva cor de rosa (Cassia grandis), nativa das florestas amazônicas do Brasil e de países vizinhos. As últimas três espécies foram adaptadas às zonas ambientais urbana e rural, abrangendo áreas do norte ao sudeste do Brasil. Na cidade de São Paulo, parque Ibirapuera, análise da sazonalidade de Cryptococcus gattii revelou a presença de propágulos de C.gattii durante o período de floração de Eucalyptus camaldulensis em duas ocasiões em dois anos nos meses de novembro, em uma das árvores nativas (Caesalpinia peltophoroides) originaria da Mata Atlântica e denominada "sibipiruna", em linguagem indígena, foi isolado C.gattii (MONTENEGRO; PAULA, 2000).

C. gattii foi isolado de amostras obtidas de partes ocas de árvores vivas: Oitis ou "árvore de chuva cor de rosa" (Moquilea tomentosa); Cássia (Cassia grandis) e Figueira (Ficus microcarpa), de restos de madeira, flores e folhas de Eucalyptus camaldulensis, em uma praça no campo da EMBRAPA localizado na cidade de Teresina, estado do Piauí, região nordeste do Brasil (LAZÉRA et al., 1998). Em região endêmica do nordeste do Brasil foram isolados C. neoformans e C. gattii em seis $(18,5 \%)$ das amostras obtidas de 32 árvores analisadas do habitat natural de C.gattii em madeira em decomposição de partes ocas de árvores vivas (LAZÉRA et al., 2000). Em áreas urbanas e rurais no nordeste do Brasil, $C$. gattii foi identificado em árvores das espécies Moquilea tomentosa, Ficus microcarpa e Cassia grandis e na região norte, floresta amazônica, foram isolados $C$. gattii e $C$. neoformans de árvores das espécies Miroxylon peruiferum e Theobroma cacao (RESTREPO et al., 2000). O primeiro relato de isolamento de $C$. neoformans var. neoformans sorotipo D de árvores do gênero Eucalyptus ocorreu no estado do Rio
Grande do Sul, no Brasil, onde a maioria dos isolados estão relacionados a $C$. gattii do sorotipo B (RIBEIRO et al., 2006). Enquanto a maioria dos isolados ambientais de $C$. gattii têm sido originados de eucaliptos, na América do Sul, o sorotipo B foi encontrado no Brasil em nichos ambientais distintos daqueles estabelecidos, tais como: (i) ninho de vespas, no Uruguai; (ii) excremento de morcegos encontrado numa casa velha e, (iii) em madeira em decomposição do tronco de Moquilea tomentosa, no Brasil (SORREL, 2001).

Amostras positivas têm sido obtidas de eucaliptos das espécies E. blakelyi, E. rudis (grupo red gum) encontradas na Índia, nos EUA, no Brasil e na Itália (ELLIS; PFEIFFER, 1994; CHAKRABARTI et al., 1997; MONTAGNA et al., 1997; MONTENEGRO; PAULA, 2000). No entanto, casos de criptococose têm sido relatados em países onde os eucaliptos não tenham se adaptado bem ou não sejam endêmicos, por exemplo, na Malásia. Além disso, mesmo com a existência de extensa pesquisa de amostragem ambiental, C. gattii não tem sido isolado de material de eucalipto em áreas com infecção endêmica, incluindo África Central, África do Sul e Papua Nova Guiné (SORREL, 2001).

A prevalência de criptococose causada por C. gattii é elevada em localizações restritas, geograficamente, às áreas tropicais e subtropicais, incluindo: Austrália, Brasil, Camboja, Havaí, sul do estado da Califórnia (EUA), México, Paraguai, Tailândia, Vietnam, Nepal e África Central. Nessas áreas $C$. gattii apresentou mais de $35 \%$ dos isolados que correspondiam ao sorotipo $\mathrm{B}$, exceto no sul do estado da Califórnia (EUA), onde 11 casos de 30 pertenciam ao sorotipo C (KWON-CHUNG; BENNETT, 1984). C. gattii é predominantemente patogênico, pode ser observado em pacientes sem imunossupressão aparente e poucos casos têm sido descritos em pacientes com AIDS, no Brasil (MELO; LACAZ; CHARBELL, 1993; ROZENBAUM; RIOS-GONÇALVES, 1994; MONTENEGRO; PAULA, 2000). As infecções causadas por $C$. gattii requerem tratamento 
agressivo e prolongado e são mais refratárias à quimioterapia antifúngica (KWON-CHUNG; BENNETT, 1992; SPEED; DUNT, 1995; SORREL, 2001). Por outro lado a incidência de C. neoformans var. grubii e C. neoformans var. neoformans tem aumentado, continuamente, devido ao crescente uso de medicamentos imunossupressores e à epidemia de AIDS, sendo considerada a segunda causa de infecção oportunista mais comum (MELO; LACAZ; CHARBELL, 1993; BUCHANAN; MURPHY, 1998; FRANZOT; SALKIN; CASADEVALL, 1999).

Na cidade de São Paulo, a criptococose causada por C. neoformans é a manifestação presente em 6,7\% dos pacientes com AIDS (MONTENEGRO; PAULA, 2000). No Brasil, de acordo com dados oficiais, $4,5 \%$ das infecções oportunistas relacionadas com AIDS são causadas por $C$. neoformans e $C$. gattii e reconhecidos como agentes etiológicos de criptococose (MINISTÉRIO DA SAÚDE, 1999). A criptococose sistêmica é considerada endêmica na região nordeste do país, $C$. gattii sorotipo B foi diagnosticado em $71,1 \%$ a $75 \%$ dos pacientes com neurocriptococose e C. neoformans em 28,9\% dos casos (CAVALCANTI, 1997; LAZÉRA et al., 2000; NISHIKAWA et al., 2003). No estado do Rio Grande do Sul entre os anos de 2000 a 2004 havia 394 casos de criptococose registrados (SECRETARIA ESTADUAL DA SAÚDE DO RIO GRANDE DO SUL, 2005).

O município de Londrina está localizado no norte do Estado do Paraná e, segundo o censo 2010, apresenta 506.701 habitantes. A ocorrência de criptococose apresentou um aumento do número de casos em humanos e em animais das espécies canina e felina, em Londrina e em outras cidades do Paraná, nos últimos oito anos. A criptococose não é considerada doença de notificação obrigatória pelo Ministério da Saúde, portanto, inexistem dados estatísticos sobre sua incidência (JORNAL DE LONDRINA, 2007; 2008b; 2008c; 2009b). Este estudo é o primeiro elaborado no município de Londrina onde há relatos efetuados pela imprensa local de casos de criptococose em humanos, porém, não foi possível obter os registros dos óbitos relacionados com a criptococose sistêmica (JORNAL DE LONDRINA, 2007; 2008a; 2008b; 2008c; 2009a; 2009b). Em humanos a AIDS é considerada doença com causa mortis de notificação obrigatória e quando a criptococose está associada a AIDS esse registro fica vinculado à doença de base. Nos últimos anos a população de pombos aumentou, consideravelmente, na cidade de Londrina devido à oferta de alimento, abrigo e falta de predadores naturais. A presença dessas aves e a falta de remoção adequada desses excrementos resultaram com o passar do tempo em acúmulo intermitente no meio ambiente urbano (JORNAL DE LONDRINA, 2008a; 2009b).

No Hospital Veterinário da Universidade Estadual de Londrina, PR, há registros de casos de criptococose sistêmica em animais domésticos representados por: cães, gatos e avestruzes, consideradas aves exóticas no Brasil (YAMAMURA et al., 2003).

\section{Conclusão}

Os fungos patogênicos das espécies $C$. neoformans e $C$. gattii foram isolados do meio ambiente representado pelo solo de locais em que há grande movimentação de transeuntes na região central da cidade de Londrina, como parques, praças e ruas. Esses fungos foram isolados do solo com excrementos de pombos e matéria vegetal próxima às árvores em locais desabrigados e expostos a chuvas constantes e luz solar. Os resultados das análises do meio ambiente confirmaram a ocorrência das espécies patogênicas Cryptococcus neoformans e Cryptococcus gattii nos ambientes onde humanos e animais podem ficar expostos aos propágulos dos fungos provenientes dos nichos ambientais. 


\section{Agradecimentos}

Agradecemos à funcionária técnica do Laboratório de Micologia Veterinária do Departamento de Medicina Veterinária Preventiva, Eliana Célia Pereira, pelo processamento das amostras e preparo das culturas micológicas. Agradecemos à Universidade Estadual de Londrina por possibilitar o meio de transporte adequado para a colheita das amostras em locais públicos da cidade de Londrina, PR.

\section{Referências}

BAUWENS, L.; SWINNE, D.; De ROEY, C. de; De MEURICH, W. Isolation of Cryptococcus neoformans var. neoformans in aviaries of the Antwerp Zoological Garden. Mycosen, Berlim, v. 29, n. 1, p. 291-294, 1986.

BOEKHOUT, T.; THEELAN, B.; DIAZ, M.; FELL, J. W.; HOP, W. C. J.; ABEL, E. C. A.; DROMER, F.; MEYER, W. Hybrid genotypes in the pathogenic yeast Cryptococcus neoformans. Microbiology, v. 147, n. 4, p. 891-907, 2001.

BUCHANAN, K. L.; MURPHY, J. W. What makes Cryptococcus neoformans a pathogen? Emerging Infectious Diseases, v. 4, n. 1, p. 1-15, 1998.

CAFARCHIA, C.; ROMITO, D.; IATTA, R.; CAMARDA, M.; MONTAGNA, M. T.; OTRANTO, D. Role of birds of prey as carriers and spreaders of Cryptococcus neoformans and other zoonotic yeasts. Medical Mycology, Abingdon, v. 44, n. 6, p. 485-492, 2006.

CAVALCANTI, M. A. S. Criptococose e seu agente no Meio Norte, estados do Piauí e Maranhão, Brasil. 1997. Tese (Doutorado em Saúde Pública) - Fundação Instituto Oswaldo Cruz. Universidade Federal do Piauí, Piauí.

CHAKRABARTI, A.; JATANA, M.; KUMAR, P.; CHATA, L.; KAUSHAL, A.; PADHYE, A. A. Isolation of Cryptococcus neoformans var. gattii from Eucalyptus camaldulensis in India. Journal of Clinical Microbiology, Washington, v. 35, n. 12, p. 3340-3342, 1997.

CURRIE, B. P.; FREUNDLICH, L. F.; CASADEVALL, A. Restriction fragment length polymorphism analysis of Cryptococcus neoformans isolates from environmental (pigeon excreta) and clinical sources in New York City. Journal of Clinical Microbiology, Washington, v. 32, n. 5, p. 1188-1192, 1994.
DELGADO, A. C.; TAGUCHI, H.; MIKAMI, Y.; MYIAJY, M.; VILLARES, M. C. B. Human Cryptococcosis: relationship of environmental and clinical strains of Cryptococcus neoformans var. neoformans from urban and rural areas. Mycopathologia, Netherlands, v. 159, n. 1, p. 7-11, 2005.

ELLIS, D.; PFEIFFER, T. Cryptococcosis and the ecology of Cryptococcus neoformans. Japanese Journal of Medical Mycology, v. 35, n. 1, p. 111-122, 1994.

. Natural habitat of Cryptococcus neoformans var. gattii. Journal of Clinical Microbiology, Washington, v. 28, n. 7, p. 1642-1644, 1990.

FRANZOT, S. P.; HAMDAN, J. S.; CURRIE, B. P.; CASDEVALL, A. Molecular epidemiology of Cryptococcus neoformans in Brazil and the United States: evidence for both local genetic differences and a global clonal population structure. Journal of Clinical Microbiology, Washington, v. 35, n. 9, p. 2243-2251, 1997.

FRANZOT, S. P.; SALKIN, I. F.; CASADEVALL, A. Cryptococcus neoformans var. grubii: separate varietal status for Cryptococcus neoformans serotype A isolates. Journal of Clinical Microbiology, Washington, v. 37, n. 3, p. 838-840, 1999.

GUGNANI, H. C.; MITCHELL, T. G.; LITVINTSEVA, A. P.; LENGELER, K. B.; HEITTMAN, J.; KUMAR, A.; BASU, S.; PALIWAL-JOSHI, A. Isolation of Cryptococcus gattii and Cryptococcus neoformans var. grubii from flowers and bark of Eucalyptus trees in India. Medical Mycology, Abingdon, v. 43, n. 6, p. 565-569, 2005.

ISHAQ, C. M.; BULMER, G. S.; FELTON, F. G. An evaluation of various environmental factors affecting the propagation of Cryptococcus neoformans. Mycopathologia and Mycology Applied, v. 35, n. 1, p. 81-90, 1968.

JORNAL DE LONDRINA. Saúde: UEL pesquisa fungos da criptococose. Londrina, p. 5, 18 de junho de 2009b.

. Criptococose: secretário diz que não há como ligar óbito a fezes de pombo. Londrina, p. 5, 2 de junho de 2009a.

Não há solução para os pombos, diz SEMA. Londrina, p. 4, 15 de janeiro de 2008c.

. Criptococose: paciente continua longe da cura. Londrina, p. 5, 13 de janeiro de 2008b.

. Doença do pombo já fez nova vítima fatal em Londrina. Londrina, p.4, 13 de janeiro de 2008a.

. Fezes de pombo deixam dois doentes na região.

Londrina, p. 4, 18 de fevereiro de 2007. 
KWON-CHUNG, K. J.; BENNETT, J. E. Cryptococcosis. In: KWON-CHUNG, K. J.; BENNETT, J. E. Medical mycology. Philadelphia: Lea \& Febiger, 1992. p. 397445.

High prevalence of Cryptococcus neoformans var. gattii in tropical and subtropical regions. $Z b l$ Bakteriologie Mikrobiologie Hygienen, v. 258, n. 2, p. 213-218, 1984.

KWON-CHUNG, K. J.; BOEKHOUT, T.; FELL, J. W.; DIAZ, M. Proposal to conserve the name Cryptococcus gattii against $C$. hondurianus and $C$. bacillisporus (Basidiomycota, Hymenomycetes, Tremellomycetidae). Táxon, v. 51, n. 9, p. 804-806, 2002.

KWON-CHUNG, K. J.; SORREL, T. C.; DROMER, F.; FUNG, E.; LEVITZ, S. M. Cryptococcosis: clinical and biological aspects. Medical Mycology, Abingdon, v. 38, p. 205-213, 2000. Suplemento 1.

LACAZ, C. S.; PORTO, E.; HEINS-VACCARI, E. M.; MELO, N. T. de. Guia para identificação: fungos, actinomicetos, algas de interesse médico. São Paulo: Sarvier, 1998. $445 \mathrm{p}$.

LACAZ, C. S.; PORTO, E.; MARTINS, J. E. C.; HEINSVACCARI, E. M.; MELO, N. T. de. Tratado de Micologia Médica Lacaz. 9. ed. São Paulo: Sarvier, 2002. 1104 p.

LAZÉRA, M. S.; CAVALCANTI, M. A.; TRILLES, L.; NISHIKAWA, M. M.; WANKE, B. Cryptococcus neoformans var.gattii: evidence for a natural habitat related to decaying wood in a pottery tree hollow. Medical Mycology, Edinburgh, v. 36, n. 2, p. 119-122, 1998.

LAZÉRA, M. S.; PIRES, F. D. A.; CAMELLOCOURA, L.; NISHIKAWA, M. M.; BEZERRA, C. C. F.; TRILLES, B. Natural habitat of Cryptococcus neoformans var. neoformans in decaying wood forming hollows in living trees. Journal of Medical Veterinary Mycology, Edinburgh, v. 34, n. 2, p. 127-131, 1996.

LAZÉRA, M. S.; SALMITO CAVALCANTI, M. A.; LONDERO, A. T.; TRILLES, B.; NISHIKAWA, M. M.; WANKE, B. Possible primary ecological niche of Cryptococcus neoformans. Medical Mycology, Abingdon, v. 38, n. 5, p. 379-383, 2000.

LAZÉRA, M. S.; WANKE, B.; NISHIKAWA, M. M. Isolation of both varieties of Cryptococcus neoformans from saprophyte sources in the city of Rio de Janeiro, Brazil. Journal of Medical Veterinary Mycology, Abingdon, v. 31, n. 6, p. 449-454, 1993.

MELO, N. T.; LACAZ, C. S.; CHARBEL, C. E. Quimiotipagem de Cryptococcus neoformans. Revisão de Literatura. Novos dados epidemiológicos sobre a criptococose. Nossa experiência com o emprego do meio de C.G.B. no estudo daquela levedura. Revista do Instituto de Medicina Tropical de São Paulo, São Paulo, v. 35, n. 5, p. 469-478, 1993.

MINISTÉRIO DA SAÚDE. Programa nacional de doenças sexualmente transmissíveis, Brasília, Brasil. Boletim Epidemiológico de AIDS, v. 1, n. 2, p. 44, 1999.

MITCHELL, T. G.; PERFECT, J. R. Cryptococcosis in the era of AIDS -100 years after the discovery of Cryptococcus neoformans. Clinical Microbiology Reviews, v. 8, n. 4, p. 515-548, 1995.

MONTAGNA, M. T.; TORTORANO, A. M.; FIORE, L.; INGLETTI, A. M.; BARBUTI, S. Cryptococcus neoformans var. gattii em Italie. Note I. Premier Casautochtone de meningite à sérotype $\mathrm{B}$ chez um sujet VIH positif. Journel de Mycologie Médicale, Paris, v. 7, n. 1, p. 9220-9225, 1997.

MONTENEGRO, H.; PAULA, C. R. Environmental isolation of Cryptococcus neoformans var. gattii and $C$. neoformans var. neoformans in the city of São Paulo, Brazil. Medical Mycology, Abingdon, v. 38, n. 5, p. 385390, 2000.

NISHIKAWA, M. M.; LAZÉRA, M. S.; BARBOSA, G. G.; TRILLES, L.; BALASSIANO, B. R.; MACEDO, R. C. L.; BEZERRA, C. C. F.; PÉREZ, M. A.; CARDARELLI, P.; WANKE, B. Serotyping of 467 Cryptococcus neoformans isolates from clinical and environmental sources in Brazil: analysis of host and regional patterns. Journal of Clinical and Microbiology, Washington, v. 41, n. 1, p. 73-77, 2003.

PFEIFFER, M.; ELLIS, D. Environmental isolation of Cryptococcus neoformans var. gattii from Eucalyptus tereticornis. Journal of Veterinary Mycology, Abingdon, v. 30, n. 5, p. 407-408, 1992.

PREFEITURA DO MUNICÍPIO DE LONDRINA. Portal da prefeitura do município de Londrina - a cidade. Disponível em: https//www. Londrina, pr.gov.br/índex. Acesso em: 02 abr. 2010.

RANDHAWA, H. S.; KOWSHIK, T.; KHAN, Z. U. Decayed wood of Syzygium cunini and Ficus religiosa living trees in Delhi/New Delhi metropolitan area as natural habitat of Cryptococcus neoformans. Medical Mycology, Abingdon, v. 41, n. 3, p. 199-209, 2003.

Efficacy of swabbing versus a conventional technique for isolation of Cryptococcus neoformans from decayed wood in tree trunk hollows. Medical Mycology, Abingdon, v. 43, n. 1, p. 67-71, 2005. 
RANDHAWA, H. S.; KOWSHIK, T.; PREETI SINHA, K.; ANVRADHA CHOWDHARY; KHAN, Z. U. ZHUNYAN; JIANPING XU; AMIT KUMAR Distribution of Cryptococcus gattii and Cryptococcus neoformans in decayed trunk wood of Syzygium cumini trees in north-western India. Medical Mycology, Abingdon, v. 44, n. 7, p. 623-630, 2006.

RESTREPO, A.; BAUMGARDNER, D. J.; BAGAGLI, E.; COOPER JR, C. R.; MCGINNIS, M. R.; LAZÉRA, M. S.; BARBOSA, F. H.; BOSCO, S. M. G.; ZOILO, Z. P. F. R.; COELHO, K. I. R.; FORTES, S. T.; FRANCO, M.; MONTENEGRO, M. R.; SANO, A.; WANKE, B. Clues to the presence of pathogenic fungi in certain environments. Medical Mycology, Abingdon, v. 38, p. 167-177, 2000. Suplemento 1.

RIBEIRO, A. M.; SILVA, L. K. R. E.; SCHRANK, I. S.; SCHRANK, A.; MEYER, MEYER, W.; VAINSTEIN, M. H. Isolation of Cryptococcus neoformans var. neoformans serotype D from Eucalypts in South Brazil. Medical Mycology, Abingdon, v. 44, n. 8, p. 707-713, 2006.

ROZENBAUM, R.; GONÇALVES, A. J. R. Clinical epidemiological study of 171 cases of Cryptococcosis. Clinical Infectious Diseases, Chicago, v. 18, n. 3, p. 369380, 1994.

SECRETARIA ESTADUAL DA SAÚDE DO RIO GRANDE DO SUL. Divisão de controle de doenças transmissíveis agudas. Porto Alegre, RS: [s. n], 2005. (Nota Epidemiológica).
SORREL, T. C. Cryptococcus neoformans variety gattii. Medical Mycology, Abingdon, v. 39, n. 2, p. 155-168, 2001.

SPEED, B.; DUNT, D. Clinical and host differences between infections with the two varieties of Cryptococcus neoformans. Clinical Infectious Diseases, Chicago, v. 21, n. 1, p. 28-34, 1995.

STAIB, F.; THIELKE, C.; RANDHAWA, H. S.; SENSKA, M.; KULINS, G. Colonization of dead plants by Cryptococcus neoformans. Zbl bakt Hyg I Abt Orig A, v. 225 , n. 3 , p. 113-124, 1972.

VARMA, A.; SWINNE, D.; STAIB, F.; BENNETT, J. E.; KWON-CHUNG, K. J. Diversity of DNA fingerprinting the infectious fungi. Journal of Clinical Microbiology, Washington, v. 33, n. 7, p. 1807-1814, 1995.

YAMAMOTO, Y.; KOHNO, S.; KOGA, H.; HAKEYA, H.; TOMONO, K.; KAKU, M.; YAMAZAKI, T.; ARIZAWA, M.; IHARA, K. Random amplified polymorphic DNA analysis of clinically and environmentally isolated Cryptococcus neoformans in Nagasaki. Journal of Clinical Microbiology, Washington, v. 33, n. 12, p. 3328-3332, 1995.

YAMAMURA, A. A. M.; MAFFEI, M. C. L.; REIS, A. C. F.; TUBELIS, P.; COSENZA, M. Infecção pulmonar por Cryptococcus neoformans var. neoformans em avestruz adulto (Struthio camelus). In: CONGRESSO BRASILEIRO DE MICROBIOLOGIA, 22., 2003. Florianópolis. Anais... Florianópolis [s.n], 2003. p. 225. 
